\title{
Prognostic impact and timing considerations for allogeneic hematopoietic stem cell transplantation in chronic myelomonocytic leukemia
}

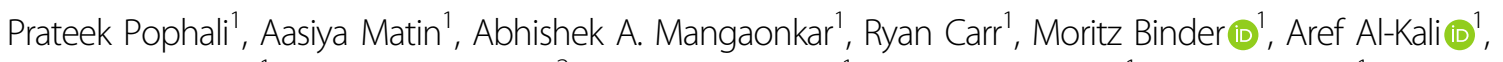 \\ Kebede H. Begna', Kaaren K. Reichard², Hassan Alkhateeb', Mithun V. Shah @1', Ayalew Tefferi', William J. Hogan (1)', \\ Mark R. Litzow ${ }^{1}$ and Mrinal M. Patnaik (1)
}

\section{Dear Editor,}

Chronic myelomonocytic leukemia (CMML) is a clonal disorder of aging hematopoietic stem cells characterized by overlapping features of myeloproliferation and myelodysplasia ${ }^{1}$, with a median overall survival (OS) of $\leq 36$ months $^{1,2}$. Hypomethylating agents (HMAs) have an overall response rate of about $30-40 \%$; however, these agents are ineffective in altering the natural disease biology due to inability to prevent acquisition of molecular abnormalities and transformation to acute myeloid leukemia $(\mathrm{AML})^{3}$. Allogeneic hematopoietic stem cell transplantation (alloHCT) is a potentially curative option, with $20-50 \%$ patients achieving long-term remissions. However, as the median age of presentation is 73 years, only a fraction $(<20 \%)$ of CMML patients are eligible for alloHCT ${ }^{4}$. Prognostic models such as the Mayo Prognostic Model (MPM), Mayo Molecular Model (MMM), Groupe Francais des Myelodysplasies, and the CMML-specific prognostic scoring system (CPSS) are important tools to identify patients at high risk for disease progression and death ${ }^{5-9}$. Several retrospective analyses of outcomes in alloHCT patients have identified adverse cytogenetics, blast percentage, HCT-comorbidity Index, time to alloHCT, disease control at the time of alloHCT and acute and chronic graft vs. host disease (GVHD) as factors influencing OS and AML-free survival (LFS) ${ }^{10-12,14,15}$.

\footnotetext{
Correspondence: Mrinal M. Patnaik (patnaik.mrinal@mayo.edu)

'Division of Hematology, Department of Medicine, Mayo Clinic, Rochester, Minnesota, USA

${ }^{2}$ Division of Hematopathology, Department of Pathology, Mayo Clinic, Rochester, Minnesota, USA
}

These authors contributed equally: Prateek Pophali, Aasiya Matin
In the absence of randomized controlled trials, the questions of optimal timing of alloHCT in CMML, prealloHCT use of HMA vs. cytotoxic chemotherapy, and the selection of patients who should be treated upfront with alloHCT remain unanswered. We performed this study to assess the outcomes and therapeutic impact of alloHCT in patients with CMML.

\section{Study population}

After Mayo Clinic Institutional Review Board approval, medical records of 406 consecutive CMML patients (age $\leq 75$ years at diagnosis) from January 1990 to December 2018 were reviewed ( 75 years being the upper age limit for alloHCT in our institution). Disease and alloHCT-related data were retrospectively collected. Next-generation sequencing (Supplementary Table 1S) for myeloid-relevant mutations was performed on bone marrow mononuclear cells at CMML diagnosis, or at first referral (within 6 months of diagnosis). Response assessment was documented as per the 2015 International Working Group (IWG) myelodysplastic/myeloproliferative neoplasm overlap neoplasm criteria $^{13}$. The CPSS, MPM, MMM, and the Mayo-French Models (MFMs) were employed for risk stratification. A 1:1 propensity score matching (PSM) analysis for age and MPM risk stratification (low, intermediate, and high risk) was used to determine the impact of alloHCT in patients who did and did not undergo alloHCT. High-risk cytogenetics included complex and monosomal karyotypes, and low risk included normal, sole $-\mathrm{Y}$, and sole der $(3 \mathrm{q})$, with the rest being included under the intermediate category per the MFM model ${ }^{9}$. Kaplan-Meier estimate of OS was computed from date of diagnosis to date of death or 
censored at last follow-up. In LFS calculation, AML replaced death as the uncensored event (details in Supplementary File under section "Supplementary Methods").

\section{Clinico-pathologic characteristics}

Seventy (17\%) CMML patients with a median age at diagnosis of 58 (range: 18-73) years underwent alloHCT at our institution; 45 (64\%) males, $46(66 \%)$ in chronic phase (CP), and 24 (34\%) after AML/blast transformation (BT) (Table 1). In the non-alloHCT (control) group, we identified 336 consecutive CMML patients with age at diagnosis $\leq 75$ [median 67 (range: 20-75)] years, 267 (79\%) patients in $\mathrm{CP}$, and 64 (19\%) with CMML that eventually had BT. The two groups were evenly matched for molecular abnormalities, except for a lower frequency of TET2 mutations in the alloHCT group (30\% vs. $56 \%$, $p=0.02$, Supplementary Table 2S).

Of the 46 patients transplanted in CP, 31 (67\%) patients received prior therapies such as HMA (48\%), AML-like induction chemotherapy (11\%), or investigational agents (7\%). Of the 24 patients transplanted in BT, $13(54 \%)$ patients received prior AML-like induction and $10(42 \%)$ received HMA followed by induction chemotherapy prior to alloHCT. There was no statistically significant difference in day 100 mortality in patients who received pretransplant HMA vs. induction therapy in both $\mathrm{CP}$ $(p=0.5)$ and BP $(p=0.1)$ CMML patients. Of the 70 CMML patients who received alloHCT, only 7 were untreated prior to receiving conditioning therapies. There was no difference in Kaplan-Meier estimate of median OS in untreated patients vs. those who received pretransplant cytoreduction (HMA, AML-like induction therapy, and investigational agents; log-rank test, $p=0.3$ ). Twenty five (57\%) and $20(87 \%)$ patients transplanted in $\mathrm{CP}$ and $\mathrm{BT}$, respectively, met criteria for complete response $(\mathrm{CR})$ or optimal marrow response at the time of alloHCT. The conditioning regimens (myeloablative vs. reduced intensity) and donor sources (matched vs. mismatched, related vs. unrelated) were evenly matched in both the CP- and BT-transplanted CMML patients (Table 1). Peripheral blood stem cells were the favored donor source in both groups ( $85 \%$ in CP vs. $87 \%$ in BT, $p=0.76$ ). Sixty six (94\%) patients had sustained donor engraftment. Similarly, rates of acute $(40 \%$ vs. $60 \%, p=0.12)$ and chronic ( $65 \%$ vs. $46 \%, p=0.24$ ) GVHD were not significantly different between CMML patients transplanted in CP and BT (Table 1). None of the transplanted patients received posttransplant HMA therapy.

\section{Survival outcomes}

At a median follow-up of 70 (95\% confidence interval (95\% CI) 27-189) months, there were 22 (31\%) deaths in the CP alloHCT group; 11 (24\%) from disease relapse, and $9(20 \%)$ from non-relapse mortality. Four $(9 \%)$ died from infections, 2 (4\%) from acute GVHD, and 2 (4\%) from multi-organ failure (1-unknown). Median OS was the higher in the CP vs. BT alloHCT group [70 $(95 \% \mathrm{CI}$ $27-189)$ vs. 32 (95\% CI 15-59) months, $p=0.001$, Supplementary Fig. 1S] and lower in non-alloHCT group [29 (95\% CI 23-35) months in CP and 22 (95\% CI 14-31) months in BT-CMML]. Post-alloHCT median OS was 67 (95\% CI 20-189) months in CP and 16 (95\% CI 7-39) months in BT-CMML ( $p=0.06$; Supplementary Fig. 2S). Median OS in transplant-eligible CMML BT patients $(\leq 75$ years old) who received alloHCT after BT was 22 months vs. 3 months in the non-alloHCT group (Supplementary Fig. 3S). Five-year OS in the post-alloHCT group was 51\% in the CP and 19\% in the BT (Supplementary Table 3S). Similar to median OS, median LFS in the non-alloHCT group was lower compared to alloHCT group [24 (95\% CI 20-28) months vs. 59 (95\% CI 27-189) months, $p=0.0001$; Supplementary Fig. 4S]. BT patients continued to fare poorly in the alloHCT group with a median LFS of 7.5 months vs. 56 months in the CP alloHCT group ( $p=0.01$, Supplementary Fig. $5 \mathrm{~S})$. At 5 years, the LFS was $47 \%$ in the CP patients who underwent alloHCT vs. only $12 \%$ who underwent alloHCT in BT (Supplementary Table 4 S). At a median follow-up of 32 (95\% CI 15-59) months in the BT alloHCT group, there were 17 (22\%) deaths: 6 (25\%) from disease relapse, 4 (13\%) due to GVHD ( 2 each from acute and chronic GVHD), 5 (21\%) due to infection, and 2 (8\%) from multi-organ failure (Supplementary Tables 5S and 6S).

Among the $\mathrm{CP}$ alloHCT recipients, median OS was not reached in the CPSS intermediate 1 (95\% CI 3-NR) and 2 (95\% CI 21-NR) risk groups, and was 12 (95\% CI 2-67, $p=0.02$ ) months in the high-risk group (Supplementary Fig. 6S). Likewise, post-alloHCT median OS was not reached in the intermediate- and low-risk MPM categories in CP patients, vs. a post-alloHCT median OS of 36 (95\% CI 8-189) months in high-risk patients (Supplementary Fig. 7S). These posttransplant OS trends were mirrored when $\mathrm{CP}$ patients were risk stratified as per the MFM (Supplementary Fig. 8S).

The composite end point of median GVHD-free/ relapse-free survival was 3.5 (95\% CI 2-7) and 7 (95\% CI $5-21)$ months in the $\mathrm{BT}$ and $\mathrm{CP}$, respectively $[p=0.02$, Supplementary Table 7S]. Further, LFS and OS in the alloHCT group did not differ in CMML alloHCT patients with vs. without chronic GVHD (Supplementary Table $8 \mathrm{~S})$. In the transplant eligible (age $\leq 75$ years) group $(n=$ 406), 200 (49\%) patients were classified as proliferative CMML, whereas 203 (50\%) were classified as dysplastic CMML (information not available for 3 patients). The Kaplan-Meier estimate median OS in the proliferative subtype was significantly lower when compared to the dysplastic subtype (20 vs. 32 months, $\log$-rank $p<0.001$ ). In the proliferative subtype, the median OS for transplant 
Table 1 Table describing clinico-pathologic and genetic characteristics of chronic myelomonocytic leukemia treated with allogeneic hematopoietic stem cell transplant.

\begin{tabular}{|c|c|c|c|c|}
\hline Variables & $\begin{array}{l}\text { All CMML patients who } \\
\text { underwent } \mathrm{HCT} \\
(n=70)\end{array}$ & $\begin{array}{l}\text { CMML patients who } \\
\text { underwent HCT in chronic } \\
\text { phase }(n=46)\end{array}$ & $\begin{array}{l}\text { CMML patients who underwent } \\
\text { HCT after blast transformation } \\
(n=24)\end{array}$ & $P$-value \\
\hline Age in years; median (range) & $58(18-73)$ & $58(26-72)$ & $57(18-73)$ & 0.24 \\
\hline Sex (Male); $n(\%)$ & $45(64)$ & $31(67)$ & $14(58)$ & 0.45 \\
\hline Hemoglobin $\mathrm{g} / \mathrm{dL}$; median (range) & $9.5(6.4-12.5)$ & $9.1(6.4-12.5)$ & $10(6.7-12.2)$ & 0.02 \\
\hline $\mathrm{WBC} \times 10^{9} / \mathrm{L} ;$ median (range) & $1.6(0.1-52.1)$ & $1.8(0.1-52.1)$ & $1.1(0.1-6)$ & 0.10 \\
\hline ANC $\times 10^{9} / \mathrm{L} ;$ median (range) & $1.5(0-43.2)$ & $1.4(0-43.2)$ & $1.7(0-5.6)$ & 0.83 \\
\hline Platelets $\times 10^{9} / \mathrm{L}$; median (range) & $56(7-277)$ & $47(7-194)$ & $65(18-277)$ & 0.07 \\
\hline $\begin{array}{l}\text { Palpable splenomegaly at transplant; } \\
\text { n (\%) }\end{array}$ & $9(13)$ & $5(11)$ & $4(18)$ & 0.43 \\
\hline Prior therapies; $n$ (\%) & $N=68$ & $N=44$ & $N=24$ & $<0.0001$ \\
\hline Observation/supportive care & $10(15)$ & $10(23)$ & $0(0)$ & \\
\hline HMA & $22(32)$ & $21(48)$ & $1(4)$ & \\
\hline AML-like induction chemotherapy & $18(27)$ & $5(11)$ & $13(54)$ & \\
\hline $\begin{array}{l}\text { HMA followed by induction } \\
\text { chemotherapy }\end{array}$ & $12(18)$ & $2(4)$ & $10(42)$ & \\
\hline Clinical trial & $3(4)$ & $3(7)$ & $0(0)$ & \\
\hline Other & $3(4)$ & $3(7)$ & $0(0)$ & \\
\hline Disease status at transplant; $n$ (\%) & $N=67$ & $N=44$ & $N=23$ & 0.0004 \\
\hline Complete remission & $23(34)$ & $8(18)$ & $15(65)$ & \\
\hline Marrow response & $22(33)$ & $17(39)$ & $5(22)$ & \\
\hline Stable disease & $8(12)$ & $8(18)$ & $0(0)$ & \\
\hline Disease progression & $14(21)$ & $11(25)$ & $3(13)$ & \\
\hline $\begin{array}{l}\text { Time to transplant from diagnosis in } \\
\text { months; median (range) }\end{array}$ & $5(0-44)$ & $7(0-22)$ & $7(2-44)$ & 0.33 \\
\hline $\mathrm{HCT}-\mathrm{Cl} ; n(\%)$ & $N=68$ & $N=45$ & $N=23$ & 0.30 \\
\hline Group 1 (0) & $16(24)$ & $8(18)$ & $8(35)$ & \\
\hline Group $2(1-2)$ & $18(26)$ & $13(29)$ & $5(22)$ & \\
\hline Group 3 ( $\geq 3$ ) & $34(50)$ & $24(53)$ & $10(43)$ & \\
\hline Type of transplant conditioning; $n$ (\%) & $N=68$ & $N=46$ & $N=22$ & 0.61 \\
\hline Myeloablative & $31(46)$ & $20(43)$ & $11(50)$ & \\
\hline Reduced intensity & $37(54)$ & $26(57)$ & $11(50)$ & \\
\hline Donor source; $n$ (\%) & $N=67$ & $N=45$ & $N=22$ & 0.83 \\
\hline Matched related donor & $28(42)$ & $17(38)$ & $11(50)$ & \\
\hline Mismatched related donor & $1(2)$ & $1(2)$ & $0(0)$ & \\
\hline Matched unrelated donor & $30(45)$ & $22(49)$ & $8(36)$ & \\
\hline Mismatched unrelated donor & $3(4)$ & $2(4)$ & $1(5)$ & \\
\hline Umbilical cord blood & $2(3)$ & $1(2)$ & $1(5)$ & \\
\hline
\end{tabular}


Table 1 continued

\begin{tabular}{|c|c|c|c|c|}
\hline Variables & $\begin{array}{l}\text { All CMML patients who } \\
\text { underwent } \mathrm{HCT} \\
(n=70)\end{array}$ & $\begin{array}{l}\text { CMML patients who } \\
\text { underwent HCT in chronic } \\
\text { phase }(n=46)\end{array}$ & $\begin{array}{l}\text { CMML patients who underwent } \\
\text { HCT after blast transformation } \\
(n=24)\end{array}$ & $P$-value \\
\hline Haploidentical donor & $3(4)$ & $2(4)$ & $1(5)$ & \\
\hline Graft source; $n(\%)$ & $N=68$ & $N=45$ & $N=23$ & 0.76 \\
\hline Peripheral blood & $58(85)$ & $38(85)$ & $20(87)$ & \\
\hline Bone marrow & $8(12)$ & $6(13)$ & $2(9)$ & \\
\hline Umbilical cord & $2(3)$ & $1(2)$ & $1(4)$ & \\
\hline Acute GVHD grade $2-4 ; n(\%)$ & 29/63 (46) & $17 / 43(40)$ & $12 / 20(60)$ & 0.12 \\
\hline Chronic GVHD; $n$ (\%) & $N=41$ & $N=30$ & $N=11$ & 0.24 \\
\hline Mild & $15(37)$ & $13(43)$ & $2(18)$ & \\
\hline Moderate & $14(34)$ & $10(33)$ & $4(36)$ & \\
\hline Severe & $12(29)$ & $7(23)$ & $5(45)$ & \\
\hline $\begin{array}{l}\text { GVHD-free, relapse-free survival in } \\
\text { months; median ( } 95 \% \mathrm{Cl} \text { ) }\end{array}$ & $6(5-8)$ & $7(5-21)$ & $3.5(2-7)$ & 0.02 \\
\hline $\begin{array}{l}\text { GRFS at } 100 \text { days post } \mathrm{HCT} \text { in } \\
\text { percentage }\end{array}$ & 70 & 76 & 54 & \\
\hline Posttransplant disease relapse; $n(\%)$ & $18(27)$ & $11(24)$ & $7(33)$ & 0.45 \\
\hline $\begin{array}{l}\text { Overall survival post transplant; } \\
\text { median }(95 \% \mathrm{Cl})\end{array}$ & $25(18-189)$ & $67(20-189)$ & $16(7-39)$ & 0.06 \\
\hline Deaths & 39 (56) & $22(48)$ & $17(71)$ & 0.06 \\
\hline
\end{tabular}

group was higher than the non-transplant group (50 vs. 19 months, log-rank $p<0.0001$ ). In dysplastic subtype, the median OS for transplant and non-transplant group was not significantly different (41 vs. 37 months, log-rank $p=0.5)$. The Kaplan-Meier estimate of median OS in patients with $\mathrm{CR}$ or optimal pre-transplant blast \% (defined as BM blast\% < 5) was higher than those without CR or optimal blast\% (50 vs. 27 months); however, this difference was not statistically significant (log-rank test, $p=0.2$ ).

\section{Survival analysis}

In a univariate survival analysis that included age, sex, CMML prognostic models, cytogenetic abnormalities, gene mutations, pre-alloHCT therapy, remission status at alloHCT, HCT-CI, time to alloHCT, donor types, stem cell source, human leukocute antigen matching, conditioning regimen, cytomegalovirus (CMV) status, blood group incompatibility, pre-alloHCT complete blood count $(\mathrm{CBC})$, acute and chronic GVHD, only abnormal karyotype (MFM intermediate and high risk; hazard ratio (HR) 2.63, 95\% CI 1.11-6.23, $p=0.03$ ) adversely impacted outcomes in CP-CMML patients that underwent alloHCT. In univariate analysis for post-alloHCT CPCMML LFS, abnormal karyotype (MFM intermediate and high risk; 2.78, 95\% CI 1.18-6.58, $p=0.02), \mathrm{WBC}<2 \times$ $10^{9} / \mathrm{L}$ at time of alloHCT $(1.06,95 \%$ CI 1.01-1.11, $p=$ 0.01 ), and absolute neutrophil count $(\mathrm{ANC})<1.5 \times 10^{9} / \mathrm{L}$ at the time of alloHCT $(1.08,95 \%$ CI $1.02-1.13, p=0.01)$ were adverse predictors. In the entire cohort of transplant-eligible CMML patients (including CP-CMML and BT-CMML patients), the three most common mutations were ASXL1 (55\%), TET2 (52\%), and SRSF2 (46\%). In CP-CMML patients, the frequency of these mutations were ASXL1 55\%, SRSF2 47\%, and TET2 35\%, whereas in BT-CMML patients, the frequencies were ASXL1 44\%, SRSF2 43\%, and TET2 30\%. In a univariate survival analysis, none of the mutations predicted postHCT outcomes (Supplementary Tables 9S and 10S).

On multivariate analysis, only abnormal karyotype (MFM intermediate and high risk; HR 2.73, 95\% CI 1.04-7.17, $p=0.02$ ) retained its negative prognostic impact (Supplementary Tables 9S and 10S).

\section{Propensity score matched analysis}

Forty-eight patients in alloHCT and non-alloHCT groups were matched for age and MPM using 1:1 PSM analysis. Median OS in the PSM-matched alloHCT group was higher compared to non-alloHCT group [40 months, (95\% CI 26-NR) vs. 23 months, (95\% CI 10-37), 

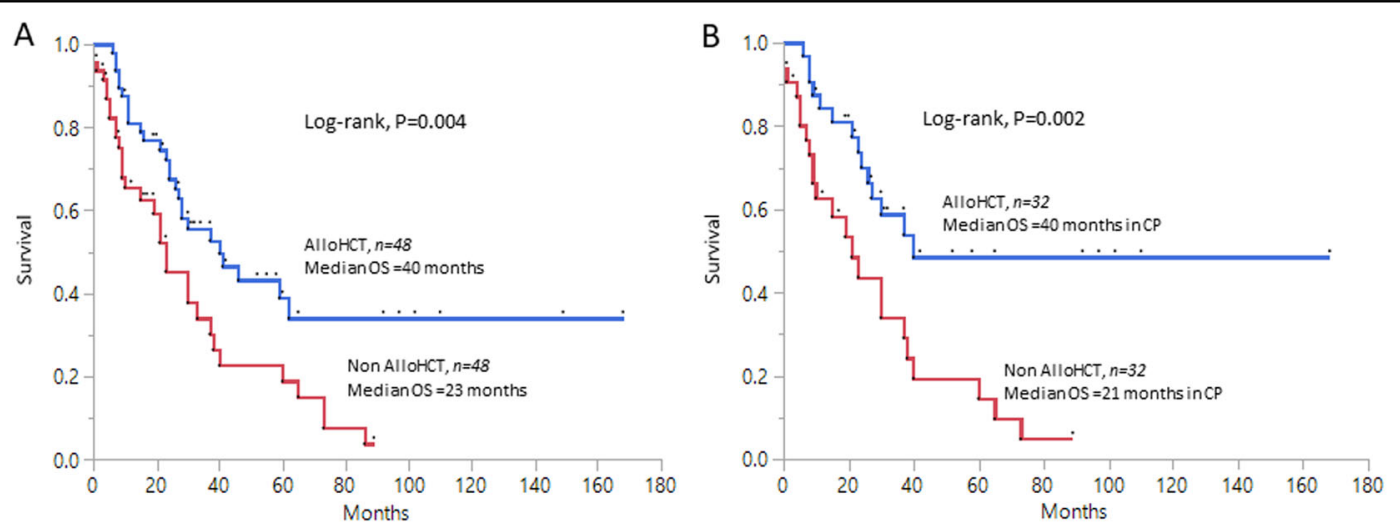

Fig. 1 Figure showing Kaplan-Meier derived overall survival curves among propensity score matched chronic myelomonocytic leukemia (CMML) cohorts. a Overall survival in a 1:1 propensity score matched cohort for age and Mayo Prognostic Model in allogeneic hematopoietic stem cell transplant (alloHCT) and non-alloHCT groups [inclusive of patients in chronic phase (CP) and blast transformation (BT)]. b Overall survival in a 1:1 propensity score matched cohort for age and Mayo Prognostic Model in alloHCT and non-alloHCT groups in CP only.

$p=0.004$, Fig. 1a]. When only CP-CMML alloHCT and non-alloHCT $(n=32)$ 1:1 matched patients were considered, the survival advantage remained significant in the alloHCT group $[40$ months, $(95 \%$ CI $26-\mathrm{NR})$ vs. 21 months (95\% CI 9-40), $p=0.002$, Fig. 1b]. Similarly, $\mathrm{CP}$ alloHCT group had a higher LFS vs. CP non-alloHCT PSM-matched group $\left[\begin{array}{llll}40 & (95 \% \text { CI } 26-N R) & \text { vs. } 20\end{array}\right.$ (95\% 9-40) months, $p=0.002]$.

In summary, within limitations of a retrospective analysis, our study confirms the survival benefit conferred by alloHCT in CMML, especially in CP disease. AlloHCT was able to achieve a 5 -year OS of 51\% in CP-CMML vs. $19 \%$ in BT-CMML, underscoring the importance of early alloHCT, especially in higher risk patients. This observation was also validated with the help of a propensity scorebased comparison (Fig. 1b). The survival advantage of alloHCT was somewhat offset by a GFRFS of only 7 months, indicating that in CMML, alloHCT can be associated with significant morbidity. We also show that intermediate to high-risk cytogenetic abnormalities by MFM are predictive of post-alloHCT relapse and inferior OS, highlighting the need for better pre-alloHCT therapies.

\section{Acknowledgements}

This publication was supported by Grant Number UL1 TR002377 from the National Center for Advancing Translational Sciences (NCATS). Its contents are solely the responsibility of the authors and do not necessarily represent the official views of the NIH.

\section{Conflict of interest}

The authors declare that they have no conflict of interest.

\section{Publisher's note}

Springer Nature remains neutral with regard to jurisdictional claims in published maps and institutional affiliations.
Supplementary Information accompanies this paper at (https://doi.org/ 10.1038/s41408-020-00387-y).

Received: 21 July 2020 Revised: 24 August 2020 Accepted: 17 September 2020

Published online: 20 November 2020

\section{References}

1. Swerdlow, S. H. et al. (eds.) WHO Classification of Tumours of Haematopoietic and Lymphoid Tissues (Revised 4th Edition) 82-86 (IARC, Lyon, 2017).

2. Arber, D. A. et al. The 2016 revision to the World Health Organization classification of myeloid neoplasms and acute leukemia. Blood 127, 2391-2405 (2016).

3. Merlevede, J. et al. Mutation allele burden remains unchanged in chronic myelomonocytic leukaemia responding to hypomethylating agents. Nat. Commun. 7, 10767 (2016).

4. Patnaik, M. M. \& Tefferi, A. Chronic myelomonocytic leukemia: 2018 update on diagnosis, risk stratification and management. Am. J. Hematol. 93, 824-840 (2018).

5. Itzykson, R. et al. Prognostic score including gene mutations in chronic myelomonocytic leukemia. J. Clin. Oncol. 31, 2428-2436 (2013).

6. Patnaik, M. M. et al. ASXL1 and SETBP1 mutations and their prognostic contribution in chronic myelomonocytic leukemia: a two-center study of 466 patients. Leukemia 28, 2206-2212 (2014).

7. Patnaik, M. M. et al. Mayo prognostic model for WHO-defined chronic myelomonocytic leukemia: ASXL1 and spliceosome component mutations and outcomes. Leukemia 27, 1504-1510 (2013).

8. Such, E. et al. Development and validation of a prognostic scoring system for patients with chronic myelomonocytic leukemia. Blood 121, 3005-3015 (2013).

9. Wassie, E. A. et al. Molecular and prognostic correlates of cytogenetic abnormalities in chronic myelomonocytic leukemia: a Mayo Clinic-French Consortium Study. Am. J. Hematol. 89, 1111-1115 (2014).

10. Eissa, $H$. et al. Allogeneic hematopoietic cell transplantation for chronic myelomonocytic leukemia: relapse-free survival is determined by karyotype and comorbidities. Biol. Blood Marrow Transplant. 17, 908-915 (2011).

11. Elliott, M. A. et al. Allogeneic stem cell transplantation and donor lymphocyte infusions for chronic myelomonocytic leukemia. Bone Marrow Transplant. 37, 1003-1008 (2006).

12. Kroger, N. et al. Allogeneic stem cell transplantation of adult chronic myelomonocytic leukaemia. A report on behalf of the Chronic Leukaemia Working Party of the European Group for Blood and Marrow Transplantation (EBMT). Br. J. Haematol. 118, 67-73 (2002) 
13. Savona, M. R. et al. An international consortium proposal of uniform response criteria for myelodysplastic/myeloproliferative neoplasms (MDS/MPN) in adults. Blood 125, 1857-1865 (2015).

14. Patnaik, M. M. et al. Blast transformation in chronic myelomonocytic leukemia: Risk factors, genetic features, survival, and treatment outcome. Am. J. Hematol. 90, 411-416 (2015)
15. Symeonidis, A. et al. Achievement of complete remission predicts outcome of allogeneic haematopoietic stem cell transplantation in patients with chronic myelomonocytic leukaemia. A study of the Chronic Malignancies Working Party of the European Group for Blood and Marrow Transplantation. Br. J. Haematol. 171, 239-246 (2015). 